\title{
Families of Periodic Solutions for Some Hamiltonian PDEs*
}

\author{
Gianni Arioli ${ }^{\dagger}$ and Hans Koch ${ }^{\ddagger}$
}

\begin{abstract}
We consider the nonlinear wave equation $u_{t t}-u_{x x}= \pm u^{3}$ and the beam equation $u_{t t}+u_{x x x x}= \pm u^{3}$ on an interval. Numerical observations indicate that time-periodic solutions for these equations are organized into structures that resemble branches and seem to undergo bifurcations. In addition to describing our observations, we prove the existence of time-periodic solutions for various periods (a set of positive measure in the case of the beam equation) along the main nontrivial "branch." Our proofs are computer-assisted.
\end{abstract}

Key words. periodic solutions, Hamiltonian PDEs, computer-assisted proof

AMS subject classifications. 35L71, 35L76

DOI. $10.1137 / 16 \mathrm{M} 1070177$

1. Introduction and main results. We prove the existence of time-periodic solutions along what looks like "branches" of solutions for two Hamiltonian partial differential equations (PDEs): the nonlinear wave equation $(\nu=1)$ and the nonlinear beam equation $(\nu=2)$ in one spatial dimension,

$$
\partial_{t}^{2} \mathfrak{u}(t, x)+(-1)^{\nu} \partial_{x}^{2 \nu} \mathfrak{u}(t, x)=f(\mathfrak{u}(t, x)), \quad(t, x) \in \mathbb{R} \times(0, \pi),
$$

with homogeneous Dirichlet $(\nu=1)$ or Navier $(\nu=2)$ boundary conditions.

This work was motivated in part by observations in a new model for suspension bridges $[3,2]$ which consists of two coupled equations: a modified nonlinear beam equation modeling the displacement of the center of the deck, and a modified nonlinear wave equation modeling the torsion of the deck. This model exhibits resonances between longitudinal and torsional modes that depend strongly on the energy (amplitudes). Thus, we are interested in families of time-periodic solutions covering a range of different amplitudes, and it is natural to consider first the simpler case of (1).

The goal is to construct solutions in a way that also yields information about their properties. The computer-assisted methods presented in this paper are well suited for such tasks. Similar techniques have been applied successfully to PDEs of elliptic and parabolic type $[32,31,24,25,30,10,9,12,13,26,6,7,5,4]$, but unlike in other constructions of periodic solutions, our differential operators are not semibounded. This leads to small denominator issues when considering parameter ranges of positive measure.

\footnotetext{
* Received by the editors April 12, 2016; accepted for publication (in revised form) by J. van den Berg August 29, 2016; published electronically January 3, 2017.

http://www.siam.org/journals/siads/16-1/M107017.html

Funding: The work of the first author was supported in part by the PRIN project "Equazioni alle derivate parziali di tipo ellittico e parabolico: aspetti geometrici, disuguaglianze collegate, e applicazioni."

${ }^{\dagger}$ Department of Mathematics, Politecnico di Milano, Piazza Leonardo da Vinci 32, 20133 Milano, Italy (gianni.arioli@polimi.it).

${ }^{\ddagger}$ Department of Mathematics, The University of Texas at Austin, Austin, TX 78712 (koch@math.utexas.edu).
} 
Initial results on time-periodic solutions for (1) covered periods that are rational multiples of $\pi$. For small-amplitude solutions, or if $f$ is near-linear, it is possible to apply perturbative methods; see e.g., $[17,18]$ and references therein. In other cases, existence results have been obtained by variational techniques $[28,27,20,22,21]$. One of the drawbacks of these techniques is that they yield very limited information about the solutions. The most recent results cover positive-measure sets of periods [14, 23, 15, 8, 11] using Nash-Moser schemes or resummation techniques (for divergent series) to deal with the problem of small denominators that arise with irrational periods. These methods are again perturbative.

Here we consider both the rational and the positive-measure case. We restrict our analysis to the specific nonlinearity $f(\mathfrak{u})=\sigma \mathfrak{u}^{3}$ with $\sigma= \pm 1$. Setting $\mathfrak{u}(t, x)=u(\alpha t, x)$, where $2 \pi / \alpha$ is the desired period for $\mathfrak{u}$, we arrive at the equation

$$
L_{\alpha} u=\sigma u^{3}, \quad L_{\alpha}=\alpha^{2} \partial_{t}^{2}+(-1)^{\nu} \partial_{x}^{2 \nu},
$$

for a function $u=u(t, x)$ that is $2 \pi$-periodic in $t$ and vanishes at $x=0, \pi$, together with its second $x$-derivative if $\nu=2$. Restricting further to solutions $u$ that are invariant under time reversal leads us to consider the vector space $\mathcal{A}^{\circ}$ of all real analytic functions $u$ on $\mathbb{R}^{2}$ that are $2 \pi$-periodic in each argument and admit a representation

$$
u=\sum_{n, k} u_{n, k} P_{n, k}, \quad P_{n, k}(t, x)=\cos (n t) \sin (k x) .
$$

We are interested mainly in solutions that are dominated by a single mode $P_{a, b}$. In the case $a=b=1$, one way to characterize such a solution is the following.

Definition 1. A solution $u$ of $(2)$ will be called a type $(1,1)$ solution if $\left|u_{n, k}\right|<\left|u_{1,1}\right|$ whenever $n>1$ or $k>1$.

Other solutions can be obtained via scaling. More specifically, if $u \in \mathcal{A}^{\mathrm{o}}$ satisfies the equation $L_{\alpha} u=\sigma u^{3}$, and if we define

$$
\tilde{u}(t, x)=b^{\nu} u(a t, b x), \quad \tilde{\alpha}=\alpha b^{\nu} / a,
$$

with $a$ and $b$ denoting nonzero integers, then $\tilde{u}$ belongs to $\mathcal{A}^{\circ}$ and satisfies $L_{\tilde{\alpha}} \tilde{u}=\sigma \tilde{u}^{3}$.

As indicated earlier, the nature of the problem (2) depends on the arithmetic properties of the frequency $\alpha$. This can be seen, e.g., from the eigenvalues of the operator $L_{\alpha}$ which are given by

$$
\lambda_{n, k}=k^{2 \nu}-(\alpha n)^{2}=\left(k^{\nu}+\alpha n\right)\left(k^{\nu}-\alpha n\right),
$$

with associated eigenfunctions $P_{n, k}$. In particular, if $\alpha$ is irrational, then all eigenvalues are nonzero, although they typically accumulate at zero if $\nu=1$.

We start by considering rational values of $\alpha$. In this case, $L_{\alpha}$ can have a nontrivial null space. We avoid this extra complication by restricting our analysis to the subspace $\mathcal{B} \subset \mathcal{A}^{\mathrm{o}}$ consisting of all functions $u \in \mathcal{A}^{\mathrm{o}}$, whose Fourier coefficients $u_{n, k}$ vanish whenever $n k$ is even. Notice that if $u$ belongs to $\mathcal{B}$, then so do $L_{\alpha} u$ and $u^{3}$. In addition, we restrict our attention to rational values of $\alpha$ that admit a representation $\alpha=p / q$, with $p$ and $q$ denoting nonzero integers of different parity (even or odd). The set of such rationals will be denoted by $\mathbb{Q}_{0}$. 
For the nonlinear wave equation $(\nu=1)$, we consider the sample set

$$
Q_{1}=\left\{\frac{3}{8}, \frac{5}{12}, \frac{7}{16}, \frac{9}{20}, \frac{13}{28}, \frac{1}{2}, \frac{15}{28}, \frac{11}{20}, \frac{9}{16}, \frac{7}{12}, \frac{5}{8}, \frac{9}{14}, \frac{11}{16}, \frac{7}{10}, \frac{13}{18}, \frac{3}{4}, \frac{11}{14}, \frac{5}{6}, \frac{7}{8}, \frac{9}{10}, \frac{11}{12}, \frac{13}{14}, \frac{17}{18}\right\} .
$$

Theorem 2. For each $\alpha \in Q_{1}$, the equation $\alpha^{2} \partial_{t}^{2} u-\partial_{x}^{2} u=u^{3}$ has a solution $u \in \mathcal{B}$ of type $(1,1)$ with $\left|u_{1,1}\right|>\sqrt{2(1-\alpha)}$.

We note that every solution $u \in \mathcal{B}$ of the equation $\alpha^{2} \partial_{t}^{2} u-\partial_{x}^{2} u=u^{3}$ with $\alpha \in \mathbb{Q}_{\text {o }}$ yields a solution $\tilde{u} \in \mathcal{B}$ of the equation $\alpha^{-2} \partial_{t}^{2} \tilde{u}-\partial_{x}^{2} \tilde{u}=-\tilde{u}^{3}$, and vice versa. The functions $u$ and $\tilde{u}$ are related via $\tilde{u}(t, x)=\alpha^{-1} u(x-\pi / 2, t-\pi / 2)$.

Our method for proving Theorem 2 applies in principle to any value $\alpha \in \mathbb{Q}_{\text {o }}$. We expect that the given bounds on $u_{1,1}$ hold for all values of $\alpha$ in a subset of $[0,1] \cap \mathbb{Q}_{0}$ whose closure has positive measure. Numerically, the curve $\alpha \mapsto u$ resembles a solution branch of the type known for finite dimensional systems; see section 2 .

Next, we consider some irrational values of $\alpha$. Here we restrict our attention to the beam equation $(\nu=2)$, where the spectrum of $L_{\alpha}$ is easier to control.

Even in this case it is difficult to construct nonsmall solutions for any specific irrational value of $\alpha$. As an example we consider a quadratic irrational $\alpha=1 / \sqrt{m}$, where $m>1$ is an integer that is not the square of an integer. In this case, Siegel's theorem on integral points on algebraic curves of genus one [19] implies that $\left|\lambda_{n, k}\right| \rightarrow \infty$ as $n$ or $k$ tends to infinity. Unfortunately we have not been able to find lower bounds on $\left|\lambda_{n, k}\right|$ that would be useful for our purpose. We sidestep this problem by making an assumption.

Theorem 3. Let $\alpha=1 / \sqrt{3}$. Assume that $\left|3 k^{4}-n^{2}\right| \geq 39$ for all $k \geq 9$ and all $n \in \mathbb{N}$. Then the equation $\alpha^{2} \partial_{t}^{2} u+\partial_{x}^{4} u=u^{3}$ has a solution $u \in \mathcal{B}$ of type $(1,1)$ with $\left|u_{1,1}\right|>1$.

We have verified the assumption $\min _{n}\left|3 k^{4}-n^{2}\right| \geq 39$ for $9 \leq k \leq 10^{12}$.

Our next result concerns irrational values of $\alpha$ that are close to the rationals in

$$
Q_{2}=\left\{\frac{1}{4}, \frac{3}{10}, \frac{9}{20}, \frac{1}{2}, \frac{7}{12}, \frac{5}{8}, \frac{3}{4}, \frac{5}{6}, \frac{7}{6}, \frac{5}{4}, \frac{19}{14}, \frac{17}{12}, \frac{31}{20}, \frac{13}{8}, \frac{31}{18}, \frac{61}{34}\right\} .
$$

Theorem 4. For each $\kappa \in Q_{2}$, there exists a set $R_{\kappa} \subset \mathbb{R}$ of positive measure that includes $\kappa$ as a Lebesgue density point, such that for each $\alpha \in R_{\kappa}$, the equation $\alpha^{2} \partial_{t}^{2} u+\partial_{x}^{4} u=\sigma u^{3}$ with $\sigma=\operatorname{sign}(1-\alpha)$ has a solution $u \in \mathcal{B}$ of type $(1,1)$ with $\left|u_{1,1}\right|>\sqrt{2|1-\alpha|}$.

Our proofs of Theorems 2-4 are computer-assisted. The general strategy and main estimates are given in section 3. This includes a definition of the sets $R_{\kappa}$ mentioned in Theorem 4 . In section 4 we show that these sets $R_{\kappa}$ have positive measure. The computer part is sketched in section 5 and is described in detail in the supplementary material (M107017_01.zip [1o$\mathrm{cal} /$ web $532 \mathrm{~KB}])$.

2. Numerical results. For numerical approximations, we truncate the Fourier series (3) for a function $u \in \mathcal{B}$ in both variables, using projections $\mathbb{E}_{N}$ and $\mathbb{P}_{K}$ defined by

$$
\mathbb{E}_{N} u=\sum_{n \leq N} \sum_{k} u_{n, k} P_{n, k}, \quad \mathbb{P}_{K} u=\sum_{n} \sum_{k \leq K} u_{n, k} P_{n, k} .
$$

However, it is useful to take into account that (2) arises from a Hamiltonian flow. The Hamiltonian $H$ is given by

$$
H(u, v)=\int_{0}^{\pi}\left[\frac{1}{2}\left(\partial_{x}^{\nu} u\right)^{2}+\frac{1}{2} \alpha^{-2} v^{2}-\frac{1}{4} \sigma u^{4}\right] d x .
$$


This Hamiltonian describes not only the full equation $L_{\alpha} u=\sigma u^{3}$ but also the truncated equation $L_{\alpha} u=\mathbb{P}_{K} \sigma u^{3}$. To be more precise, we can consider $H$ as a function on $\mathcal{B}_{K} \times \dot{\mathcal{B}}_{K}$, where $\mathcal{B}_{K}$ is the range of $\mathbb{P}_{K}$, and where $\dot{\mathcal{B}}_{K}$ is the set of all functions $\partial_{t} u$ with $u \in \mathcal{B}_{K}$. The flow defined by the Hamiltonian $H$ is given by $\partial_{t} u=\nabla_{v} H$ and $\partial_{t} v=-\nabla_{u} H$, using gradients with respect to the $\mathrm{L}^{2}$ inner product. In particular, $\partial_{t} u=\alpha^{-2} v$. Substituting $v=\alpha^{2} \partial_{t} u$ into the equation $\partial_{t} v=-\nabla_{u} H$ yields $L_{\alpha} u=\mathbb{P}_{K} \sigma u^{3}$. If we define $\mathbb{P}_{\infty}$ to be the identity operator, then the same applies to $K=\infty$.

In what follows, we always assume that $\sigma=\operatorname{sign}(1-\alpha)$.

For numerical computations, we have to truncate the equation $L_{\alpha} u=\mathbb{P}_{K} \sigma u^{3}$ further to $L_{\alpha} u=\mathbb{E}_{N} \mathbb{P}_{K} \sigma u^{3}$. This doubly truncated system is no longer Hamiltonian. But this can be remedied partly by choosing $N \gg K$ whenever necessary. The fixed point equation associated with $L_{\alpha} u=\mathbb{E}_{N} \mathbb{P}_{K} \sigma u^{3}$ is

$$
u=\mathcal{F}_{\alpha}^{N K}(u) \stackrel{\text { def }}{=} L_{\alpha}^{-1} \mathbb{E}_{N} \mathbb{P}_{K} \sigma u^{3}, \quad \sigma=\operatorname{sign}(1-\alpha) .
$$

Since $\mathcal{F}_{\alpha}^{N K}$ is a map on the finite dimensional space $\mathbb{E}_{N} \mathcal{B}_{K}$, approximate fixed points can be found by standard numerical methods.

We consider values of $\alpha$ in the interval $[0, \nu]$. By the implicit function theorem, the solutions of $\mathcal{F}_{\alpha}^{N K}(u)=u$ for which $D \mathcal{F}_{\alpha}^{N K}(u)$ has no eigenvalue 1 are organized into branches, where $u$ depends smoothly on the parameter $\alpha$. The union of all smooth branches that include a solution of type $(1,1)$ will be referred to as the $(1,1)$ branch or "main" branch. Scaling each solution on this main branch via (4) yields what we will call the $(a, b)$ branch.
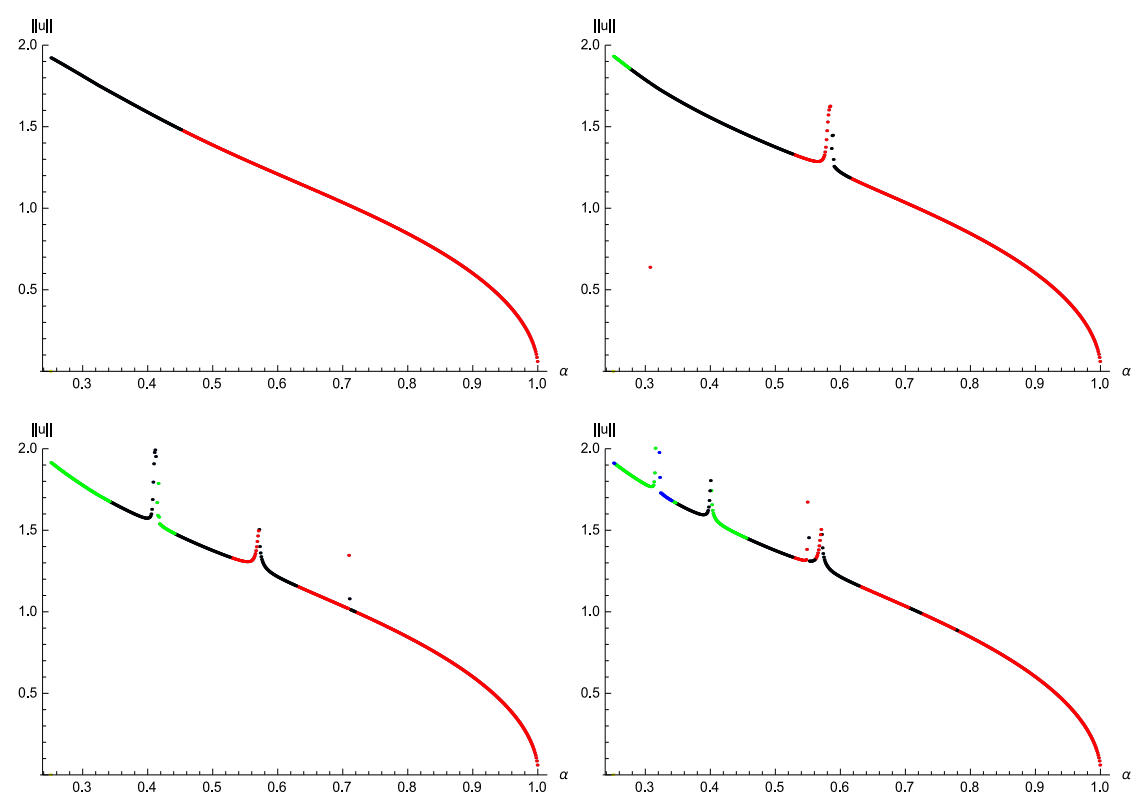

Figure 1. $(\nu=1)$ norm versus $\alpha$, for the solutions of (8) with $N=K$ and $K=3,5,7,9$.

We start with the nonlinear wave equation $(\nu=1)$. Our first observation is that the main branch covers a large fraction of the interval $[0,1]$. The four graphs in Figure 1 display 
the norm $\|u\|_{0}=\sum_{n, k}\left|u_{n, k}\right|$ as a function of $\alpha$ along the main branch, for $N=K$ and $K=3,5,7,9$.

As one would expect, the larger $K$ is, the less regular the graph. But the changes appear rather tame. Spikes appear as $K$ is increased, but they get increasingly narrower and become invisible at any given resolution. To highlight places of possible bifurcations, we use colors to indicate the index of the solution $u$, that is, the number of eigenvalues of $\operatorname{DF}_{\alpha}^{N K}(u)$ of modulus larger than 1 (red $\rightarrow 1$, black $\rightarrow 2$, green $\rightarrow 3$, blue $\rightarrow 4$ or more).

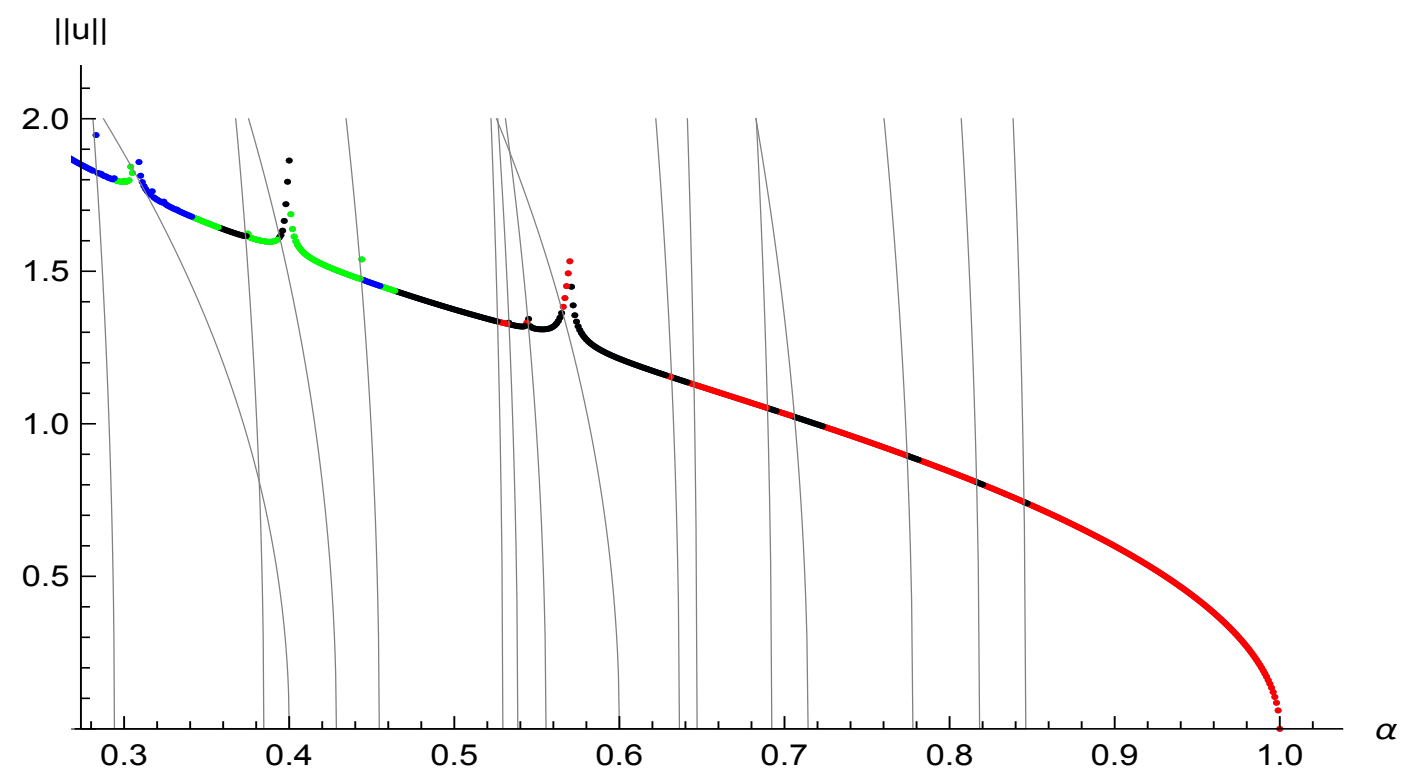

Figure 2. $(\nu=1)$ The $(1,1)$ branch and some other $(a, b)$ branches (thin lines).

Many of these bifurcations seem to involve other $(a, b)$ branches: Figure 2 shows the main branch with $K=N=59$, together with several $(a, b)$ branches (thin lines). Among the points on this branch are the solutions described in Theorem 2. The values $\alpha \in Q_{1}$ have been chosen away from the "low order" bifurcations that are visible in Figure 2. Here the branch $(a, b)$ can be identified by using that it bifurcates out of $u=0$ at $\alpha=b / a$.

We study this phenomenon in more detail for values of $\alpha$ in the interval $[0.57,0.59]$, where the first spike appears at truncation $N=K=5$. To this end we choose $K=7$, and $N \gg K$ in order to preserve (approximately) the Hamiltonian character of the equation. Figure 3 shows the values of the coefficients $u_{1,1}, u_{3,3}, u_{5,3}$ and of the norm $\|u\|_{0}$, for the solutions $u$ that we found on the given $\alpha$-interval. These graphs indicate clearly that the $(1,1)$ branch coming from higher values of $\alpha$ undergoes a fold bifurcation at $\alpha \simeq 0.571$ and then bends back until it reaches a pitchfork bifurcation at $\alpha \simeq 0.585$. The main branch at the pitchfork bifurcation is the $(5,3)$ branch, which bifurcates out of $u=0$ at $\alpha=3 / 5$. The secondary branch is the continuation of the $(1,1)$ branch.

We did not investigate any of the other bifurcations, but our guess is that all bifurcations of the $(1,1)$ branch involve one of the other $(a, b)$ branches.

Next we consider the nonlinear beam equation $(\nu=2)$. Our numerical results for this 

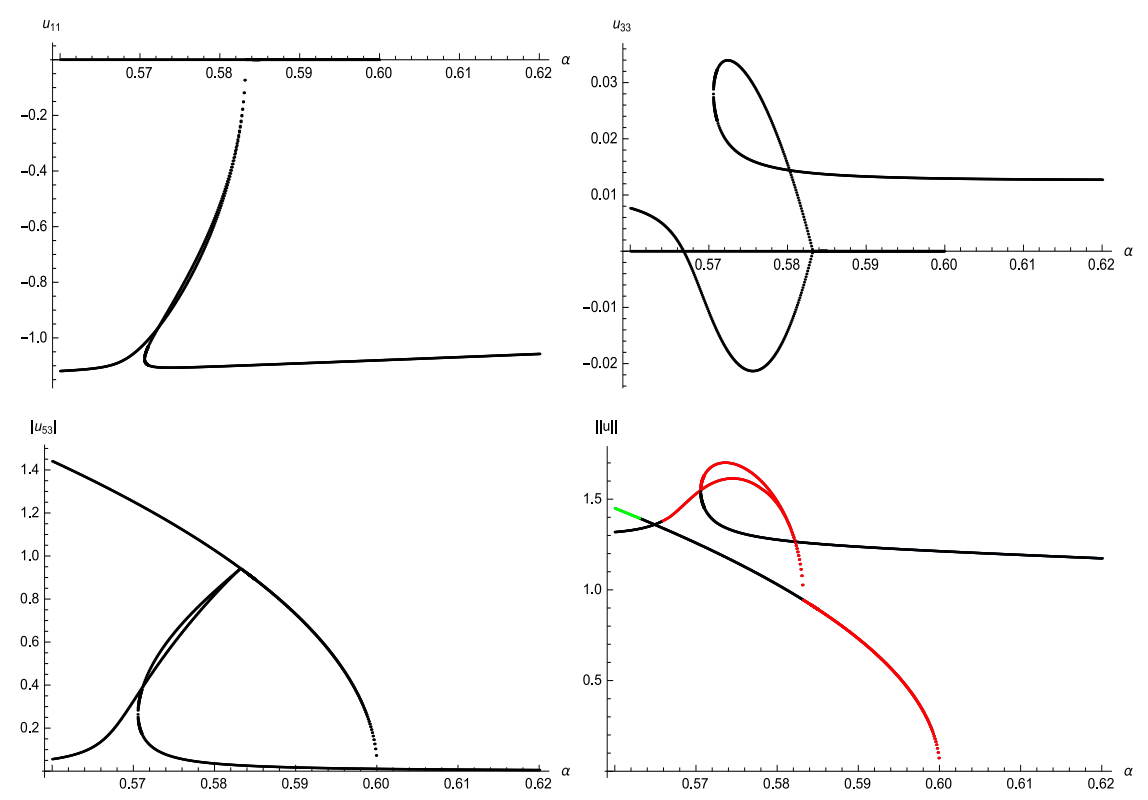

Figure 3. $(\nu=1)$ Coefficients $u_{1,1}, u_{3,3}, u_{5,3}$ and norm $\|u\|_{0}$ versus $\alpha$, for the solutions of the approximate Hamiltonian system, $N \gg K=7$, near the crossing of the $(1,1)$ branch and the $(5,3)$ branch.

equation show similarities with the above-mentioned results for the wave equation $(\nu=1)$. Here we are using $K=63$ and $N=127$. The two graphs in Figure 4 depict the $(1,1)$ branch of solutions of (8), for $\alpha<1$ (left) and for $\alpha>1$ (right). These graphs appear much more regular than our graphs for $\nu=1$.
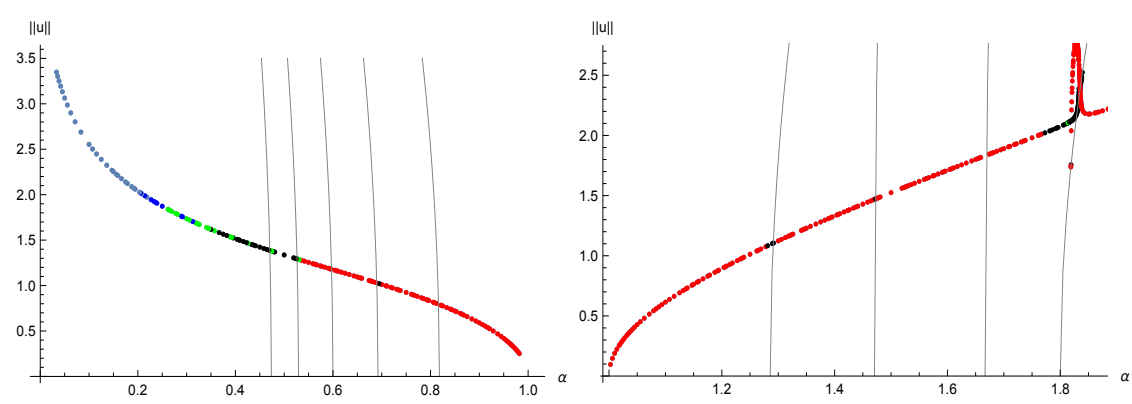

Figure 4. $(\nu=2)$ The $(1,1)$ branch for $\alpha<1$ (left) and for $\alpha>1$ (right). The thin lines represent a few other $(a, b)$ branches.

Still, we observe a crossing between the $(1,1)$ branch and the $(5,3)$ branch, with a bifurcation pattern similar to the one for the wave equation; see Figure 5, which shows an enlargement of the spike at $\alpha \simeq 1.83$. The index changes that are visible in Figure 4 indicate that similar bifurcations occur at other branch crossings as well.

The values of $\alpha$ used in Figure 4 include the values from the set $Q_{2}$ defined before Theorem 4. For these values of $\alpha$, there is no visible difference between our numerical values of the norms $\|u\|_{0}$ and our rigorous norm bounds for the true solutions of $L_{\alpha} u=\sigma u^{3}$. We have 


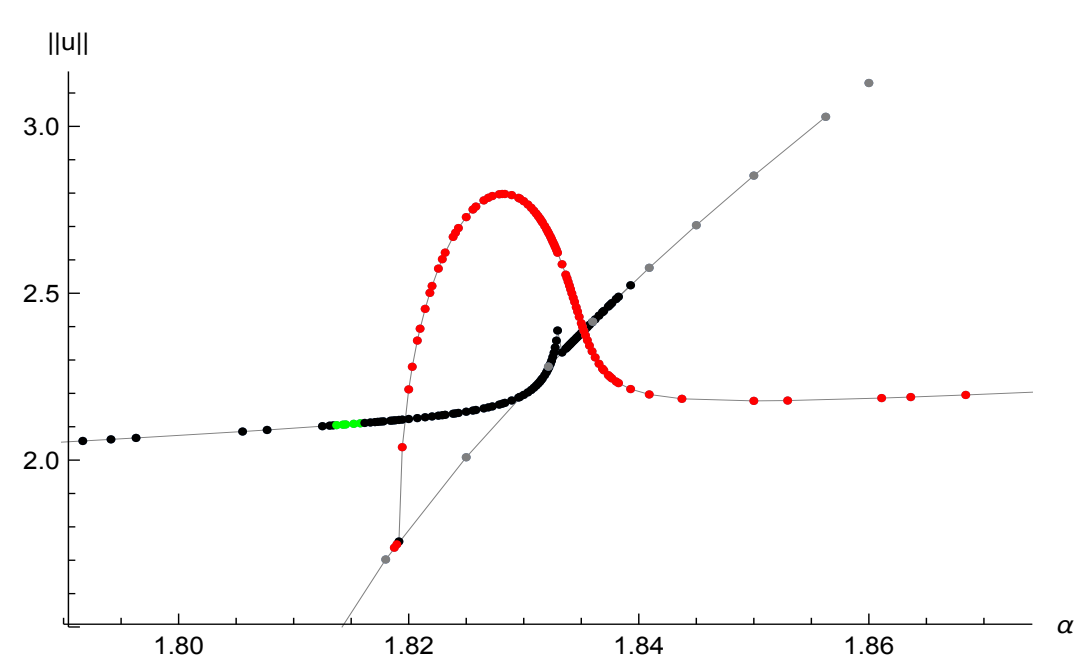

Figure 5. $(\nu=2)$ Enlargement of the spike at $\alpha \simeq 1.83$ in Figure 4 .

no doubt that the other points in Figure 4 could be verified in the same sense.

This suggests that the beam equation has families of periodic solutions that, at any finite resolution, are indistinguishable from the solution branches of a truncated beam equation. The same seems to be true for the wave equation. These families cover a wide range of periods, with varying amplitudes. The existence of such families (of longitudinal modes) was conjectured in $[3,2]$ for a model of a bridge, and we believe that our results for (1) lend support to this conjecture.

3. Estimates for linear operators. First, we reformulate our main results in terms of contraction mappings. After describing what types of estimates are needed in order to control the linear operators involved, we will give explicit bounds on the operator $L_{\alpha}$.

Given a pair $\rho=\left(\rho_{1}, \rho_{2}\right)$ of positive real numbers, denote by $\mathcal{A}_{\rho}^{\mathrm{o}}$ the closure with respect to the norm

$$
\|u\|_{\rho}=\sum_{n, k}\left|u_{n, k}\right| \varrho_{1}^{n} \varrho_{2}^{k}, \quad \varrho_{j}=1+\rho_{j},
$$

of the space of Fourier polynomials $u \in \mathcal{A}^{\mathrm{o}}$. Notice that the functions in $\mathcal{A}_{\rho}^{\mathrm{o}}$ are analytic on the domain $\mathcal{D}_{\rho}$ given by $|\operatorname{Im} t|<\ln \left(\varrho_{1}\right)$ and $|\operatorname{Im} x|<\ln \left(\varrho_{2}\right)$. In particular, $\mathcal{A}_{\rho}^{\circ}$ is a subset of $\mathcal{A}^{\mathrm{o}}$. We also define $\mathcal{B}_{\rho}=\mathcal{B} \cap \mathcal{A}_{\rho}^{\mathrm{o}}$. The operator norm of a bounded linear operator $\mathcal{L}: \mathcal{B}_{\rho} \rightarrow \mathcal{B}_{\rho}$ will be denoted by $\|\mathcal{L}\|_{\rho}$.

The domain of the operator $L_{\alpha}$ is defined to be the subspace of all functions $u \in \mathcal{B}_{\rho}$ for which the sum $\sum_{n, k}\left|\lambda_{k, n} u_{k, n}\right| \varrho_{1}^{n} \varrho_{2}^{k}$ is finite. In the cases considered here, we will show that the eigenvalues of $L_{\alpha}$ are bounded away from zero, implying that $L_{\alpha}$ has a bounded inverse on $\mathcal{B}_{\rho}$. Consequently, (2) with $\sigma=\operatorname{sign}(1-\alpha)$ can be written as

$$
u=\mathcal{F}_{\alpha}(u) \stackrel{\text { def }}{=} L_{\alpha}^{-1} \sigma u^{3}, \quad \sigma=\operatorname{sign}(1-\alpha) .
$$

In order to solve the fixed point problem for $\mathcal{F}_{\alpha}$ we consider an approximate Newton map $\mathcal{N}_{\alpha}$ associated with $\mathcal{F}_{\alpha}$. To be more specific, we first determine an approximate fixed point 
$u_{0}$ and write $u=u_{0}+A h$, where $A$ is a suitable linear isomorphism of $\mathcal{B}_{\rho}$. Then $u$ is a fixed point of $\mathcal{F}_{\alpha}$ if and only if $h$ is a fixed point of the map $\mathcal{N}_{\alpha}$ defined by

$$
\mathcal{N}_{\alpha}(h)=\mathcal{F}_{\alpha}\left(u_{0}+A h\right)-u_{0}+(\mathrm{I}-A) h .
$$

By choosing $A$ to be an approximate inverse of $\mathrm{I}-D \mathcal{F}_{\alpha}\left(u_{0}\right)$, we can expect $\mathcal{N}_{\alpha}$ to be a contraction near $u_{0}$.

Given $r>0$ and $u \in \mathcal{B}_{\rho}$, denote by $B_{r}(u)$ the open ball of radius $r$ in $\mathcal{B}_{\rho}$, centered at $u$. Theorem 4 is proved by verifying the following bounds.

Lemma 5. For each $\kappa \in Q_{2}$, there exists a set $R_{\kappa} \subset \mathbb{R}$ of positive measure that includes $\kappa$ as a Lebesgue density point, a pair $\rho$ of positive real numbers, a Fourier polynomial $u_{0} \in \mathcal{B}_{\rho}$, a linear isomorphism $A: \mathcal{B}_{\rho} \rightarrow \mathcal{B}_{\rho}$, and positive constants $K, \delta$, $\varepsilon$ satisfying $\varepsilon+K \delta<\delta$, such that for every $\alpha \in R_{\kappa}$, the map $\mathcal{N}_{\alpha}$ defined by (10) and (11) is analytic on $B_{\delta}(0)$ and satisfies

$$
\left\|\mathcal{N}_{\alpha}(0)\right\|_{\rho}<\varepsilon, \quad\left\|D \mathcal{N}_{\alpha}(h)\right\|_{\rho}<K, \quad h \in B_{\delta}(0) .
$$

This lemma, together with the contraction mapping principle, implies that for each $\kappa \in K$ and each $\alpha \in R_{\kappa}$, the map $\mathcal{N}_{\alpha}$ has a unique fixed point $h_{*} \in B_{\delta}(0)$. The corresponding function $u_{*}=u_{0}+A h_{*}$ is a fixed point of $\mathcal{F}_{\alpha}$ and thus solves (2). Thus, Lemma 5 implies Theorem 4 , after we verify that $u=u_{*}$ is of type $(1,1)$ and satisfies $\left|u_{1,1}\right|>\sqrt{2|1-\alpha|}$. Notice that $u_{*}$ belongs to the ball $B_{r}\left(u_{0}\right)$ of radius $r=\delta\|A\|_{\rho}$.

Similar contraction estimates are used to prove Theorems 2 and 3. The corresponding lemmas are completely analogous to Lemma 5 . Thus, we shall not state them explicitly. Our proof of these lemmas is computer-assisted. As a by-product we obtain accurate bounds on the solutions and related quantities.

We will now describe the main estimates involved, which are specific to the problem at hand. More "generic" aspects of the proof are described in section 5. The general strategy is to approximate a function $u \in \mathcal{A}_{\rho}^{\mathrm{o}}$ by a Fourier polynomial $P$ and to estimate the difference $\mathcal{E}=u-P$. A typical step in our proof yields much more information about the error $\mathcal{E}$ than just its norm. Keeping track of such information can significantly improve the estimates in subsequent steps.

In order to describe our choice of error terms we need to introduce the following: Given positive integers $N$ and $K$, denote by $\mathcal{A}_{\rho, N, K}^{\mathrm{o}}$ the space of all functions $u \in \mathcal{A}_{\rho}^{\mathrm{o}}$ whose Fourier coefficients $u_{n, k}$ vanish whenever $n<N$ or $k<K$. Now let $N$ and $K$ be fixed. Then we represent a function $u \in \mathcal{A}_{\rho}^{\mathrm{o}}$ as a finite sum

$$
u=P+\mathcal{E}, \quad P=\sum_{\substack{n \leq N \\ k \leq K}} c_{n, k} P_{n, k}, \quad \mathcal{E}=\sum_{\substack{n \leq 2 N \\ k \leq 2 K}} E_{n, k},
$$

where $E_{n, k}$ is a function in $\mathcal{A}_{\rho, n, k}^{\mathrm{o}}$. In this context, a bound on $u$ consists of upper and lower bounds on the coefficients $c_{n, k}$ for $n \leq N$ and $k \leq K$ and an upper bound on $\left\|E_{n, k}\right\|_{\rho}$ for $n \leq 2 N$ and $k \leq 2 K$. Notice that the representation (13) is highly nonunique. This allows for a wide range of different bounds on functions in $\mathcal{A}_{\rho}^{\mathrm{o}}$.

Our estimates on a continuous linear operator $\mathcal{L}: \mathcal{A}_{\rho}^{\mathrm{o}} \rightarrow \mathcal{A}_{\rho}^{\mathrm{o}}$ consist of a bound on $\mathcal{L} P_{n, k}$ for each $n \leq N$ and $k \leq K$, together with bounds of the form

$$
\|\mathcal{L} E\|_{\rho} \leq B_{\mathcal{L}, k, n}\|E\|_{\rho}, \quad E \in \mathcal{A}_{\rho, n, k}^{\mathrm{o}}
$$


for each $k \leq 2 K$ and $n \leq 2 N$. This also yields a bound on the operator norm on $\mathcal{L}$, namely,

$$
\|\mathcal{L}\|_{\rho} \leq\left(\bigvee_{\substack{n \leq N \\ k \leq K}} \varrho_{1}^{-n} \varrho_{2}^{-k}\left\|\mathcal{L} P_{n, k}\right\|_{\rho}\right) \vee B_{\mathcal{L}, N+1,0} \vee B_{\mathcal{L}, 0, K+1}
$$

Here and in what follows, if $s$ and $t$ are real numbers, then $s \vee t$ denotes the maximum value of $s$ and $t$. The inequality (15) is used, e.g., to verify the bound $\left\|D \mathcal{N}_{\alpha}(h)\right\|_{\rho}<K$ in Lemma 5 .

The following proposition will be useful for estimating the inverse of the operator $L_{\alpha}$.

Proposition 6. Let $s, t, \delta$ be positive real numbers. If $|t-s| \geq \delta$, then

$$
\left|t^{2}-s^{2}\right| \geq(2(s \vee t)-\delta) \delta .
$$

Proof. Fix $t>0$. For $s \in \mathbb{R}$ positive define $f(s)=\left(t^{2}-s^{2}\right)^{2}$. Then $f(s) \geq f(t)=0$. The derivative $f^{\prime}(s)=-4 s\left(t^{2}-s^{2}\right)$ is positive for $s>t$ and negative for $s<t$. Assume that $|t-s|>\delta$. Then $s$ lies outside the interval $[t-\delta, t+\delta]$. So either $f(s) \geq f(t+\delta)$ or $f(s) \geq f(t-\delta)$. But

$$
f(t \pm \delta)=(t+(t \pm \delta))^{2}(t-(t \pm \delta))^{2}=(2 t \pm \delta)^{2} \delta^{2} \geq(2 t-\delta)^{2} \delta^{2} .
$$

The same holds if $s$ and $t$ are exchanged. This proves (16).

First, we consider rational values of $\alpha$. Define $\mathcal{B}_{\rho, n, k}=\mathcal{B} \cap \mathcal{A}_{\rho, n, k}^{\mathrm{o}}$.

Proposition 7. Let $\kappa=p / q$ with $p$ odd and $q$ even. Then the operator $L_{\kappa}$ with $\nu \geq 1$ has a compact inverse $L_{\kappa}^{-1}: \mathcal{B}_{\rho} \rightarrow \mathcal{B}_{\rho}$. If $n$ and $k$ are odd, then

$$
\left\|L_{\kappa}^{-1} E\right\|_{\rho} \leq \frac{q^{2}}{2\left(q k^{\nu} \vee p n\right)-1}\|E\|_{\rho} \quad \forall E \in \mathcal{B}_{\rho, n, k} .
$$

Proof. If $u \in \mathcal{B}_{\rho}$ is a Fourier polynomial, then $L_{\kappa}^{-1} u$ is well defined, and

$$
\left(L_{\kappa}^{-1} u\right)_{n, k}=\frac{q^{2}}{\left(q k^{\nu}+p n\right)\left(q k^{\nu}-p n\right)} u_{n, k}
$$

for odd $n$ and $k$. Here we have used that $q k^{\nu}-p n$ is odd and thus nonzero. Using (16) with $t=q k^{\nu}, s=p t$, and $\delta=1$, we obtain

$$
\left|\left(L_{\kappa}^{-1} u\right)_{n, k}\right| \leq \frac{q^{2}}{2\left(q k^{\nu} \vee p n\right)-1}\left|u_{n, k}\right| .
$$

Given that the fraction on the right-hand side is a decreasing function of both $n$ and $k$, with a zero limit as $n \vee k \rightarrow \infty$, the assertion follows.

Let $\mathbb{N}_{\mathrm{o}}=\{1,3,5, \ldots\}$. For irrational $\alpha$ we need to estimate the quantities

$$
\phi(n, k)=\inf _{\substack{x \geq k \\ y \geq n}}\left|\left(\beta x^{\nu}\right)^{2}-y^{2}\right|, \quad n, k \in \mathbb{N}_{\mathrm{O}},
$$

where $\beta=\alpha^{-1}$. Here and in what follows, $x$ and $y$ always denote odd positive integers. Notice that the function $\phi: \mathbb{N}_{\mathrm{O}} \times \mathbb{N}_{\mathrm{O}} \rightarrow \mathbb{R}$ is nondecreasing in each argument.

The following proposition is trivial, but it gives an explicit expression for the bounds $B_{\mathcal{L}, k, n}$ that appear in (14) for the operator $\mathcal{L}=L_{\alpha}^{-1}$. 
Proposition 8. Assume that the function $\phi$ is strictly positive and unbounded. Then the operator $L_{\alpha}$ has a compact inverse $L_{\alpha}^{-1}: \mathcal{B}_{\rho} \rightarrow \mathcal{B}_{\rho}$. Furthermore, for any $n, k \in \mathbb{N}_{o}$,

$$
\left\|L_{\alpha}^{-1} E\right\|_{\rho} \leq \frac{\beta^{2}}{\phi(n, k)}\|E\|_{\rho} \quad \forall E \in \mathcal{B}_{\rho, n, k} .
$$

We note that the compactness of $L_{\alpha}^{-1}$ is not really needed, but just the bound (22).

Concerning the case $\beta=\sqrt{3}$, notice that the assumption in Theorem 3 says that $\phi(n, 9) \geq$ 39 for all $n$. Based on this assumption, it suffices to compute a finite number of terms $\left|3 x^{4}-y^{2}\right|$ in order to determine $\phi(n, k)$ for every $k<9$ and every $n$.

By estimating the constant $\beta^{2} / \phi(n, k)$ in $(22)$ as a function of $\alpha$, we obtain the following.

Lemma 9. There exists a set $R \subset \mathbb{R}$ of full measure such that if $\alpha \in R$, then $L_{\alpha}=\alpha^{2} \partial_{t}^{2}+\partial_{x}^{4}$ has a compact inverse $L_{\alpha}^{-1}: \mathcal{B}_{\rho} \rightarrow \mathcal{B}_{\rho}$. Let $\kappa=p / q$ with $p$ odd and $q$ even. Given odd positive integers $K$ and $N$, denote by $R_{\kappa}$ the set of all $\alpha \in R$ with the property that

$$
\left\|L_{\alpha}^{-1} E\right\|_{\rho} \leq \frac{4 p^{2} \alpha^{-2} / 7}{p\left(\alpha^{-1} k^{2} \vee n\right)-7 / 16}\|E\|_{\rho} \quad \forall E \in \mathcal{B}_{\rho, n, k}
$$

holds for all $n \leq N$ and $k \leq K$. Then $\kappa$ is a Lebesgue density point for $R_{\kappa}$.

This lemma will be proved in the next section. Notice that if $\alpha=\kappa$, then (23) follows from (18). Thus $\kappa \in R_{\kappa}$. We note that the inequality (23) is used in our programs to estimate the error terms (14) for the operator $\mathcal{L}=L_{\alpha}^{-1}$. It cannot be weakened substantially, e.g., to increase the set $R_{\kappa}$, without jeopardizing the bounds (12).

4. Irrational frequencies. Consider the operator $L_{\alpha}=\alpha^{2} \partial_{t}^{2}+\partial_{x}^{4}$ for irrational values of $\alpha$. Let $\beta=\alpha^{-1}$. The eigenvalues of $L_{\alpha}^{-1}: \mathcal{A}_{0,1}^{\mathrm{o}} \rightarrow \mathcal{A}_{0,1}^{\mathrm{o}}$ are

$$
\left(L_{\alpha}^{-1}\right)_{n, k}=\frac{\beta^{2}}{\left(\beta k^{2}+n\right)\left(\beta k^{2}-n\right)},
$$

with eigenvectors $P_{n, k}$. Here and in what follows, $n \geq 0$ and $k \geq 1$. By (16) we have

$$
\left|\left(L_{\alpha}^{-1}\right)_{k, n}\right| \leq \frac{\beta^{2}}{\left(2\left(\beta k^{2} \vee n\right)-\mid\left\lceil\beta k^{2}\right\rfloor\right)\left|\left\lceil\beta k^{2}\right\rfloor\right|},
$$

where $\|s\|=\operatorname{dist}(s, \mathbb{Z})$ denotes the distance of $s \in \mathbb{R}$ from the set of integers.

The following proposition is part of a result in [29]. We include a proof since it is short and simple. Let $\left(\psi_{1}, \psi_{2}, \psi_{3}, \ldots\right)$ be a summable sequence of nonnegative real numbers.

Proposition 10. Let $m \geq 1$ and $\Psi_{m}=\sum_{k \geq m} \psi_{k}$. Consider an interval $I=(a, a+1]$. Then the set of all $\beta \in I$ satisfying

$$
\mid\left[\beta k^{2} \|<\psi_{k} \quad \text { for some } \quad k \geq m\right.
$$

has measure at most $2 \Psi_{m}$. 
Proof. We may assume that $\psi_{k} \leq \frac{1}{2}$. Consider the circle $\mathbb{R} / \mathbb{Z}$. For simplicity we identify this circle with the interval $S=\left(-\frac{1}{2}, \frac{1}{2}\right]$. For $s \in \mathbb{R}$ define $\lceil s\rfloor$ to be the unique real number in $S$ that differs from $s$ by an integer. Notice that $\mid\left\lceil\beta k^{2}\right\rfloor<\psi_{k}$ if and only if $\left\lceil\beta k^{2}\right\rfloor$ belongs to $\left(-\psi_{k}, \psi_{k}\right)$.

The map $\beta \mapsto\left\lceil\beta k^{2}\right\rfloor$ covers the circle $S$ exactly $k^{2}$ times as $\beta$ ranges in $I$. So the set $I_{k}$ of all $\beta \in I$ that satisfies $\mid\left[\beta k^{2} \|<\psi_{k}\right.$ has measure precisely $2 \psi_{k}$. The set of all $\beta \in I$ that satisfy (26) is the union $\bigcup_{k \geq m} I_{k}$ and thus has measure at most $2 \Psi_{m}$.

This proposition implies, e.g., that for every $\varepsilon>0$ and almost every $\beta \in I$, we have $\mid\left[\beta k^{2} \| \geq k^{-1-\varepsilon}\right.$ for all but finitely many values of $k>0$. Combining this fact with the bound (25) implies the next corollary.

Corollary 11. For almost every $\alpha \in \mathbb{R}$, the operator $L_{\alpha}=\alpha^{2} \partial_{t}^{2}+\partial_{x}^{4}$ has a compact inverse $L_{\alpha}^{-1}: \mathcal{A}_{0,1}^{\mathrm{o}} \rightarrow \mathcal{A}_{0,1}^{\mathrm{o}}$.

The following extends Proposition 10 to smaller intervals, at the cost of imposing a lower bound on $m$.

Proposition 12. Consider a subinterval $J \subset I$. Assume that $m^{-2} \leq|J|$. Then the set of all $\beta \in J$ satisfying (26) has measure less than $4 \Psi_{m}|J|$.

Proof. We use the notation and intervals $I_{k}$ introduced in the proof of Proposition 10. For simplicity assume that $a=0$.

Let $\psi_{k}^{\prime}=k^{-2} \psi_{k}$. If we identify $\left(-\psi_{k}^{\prime}, 0\right]$ with $\left(1-\psi_{k}^{\prime}, 1\right]$, then $I_{k}$ is the union of the $k^{2}$ intervals $\left(n k^{-2}-\psi_{k}^{\prime}, n k^{-2}+\psi_{k}^{\prime}\right)$ for $0 \leq n<k^{2}$. Notice that these intervals are centered at integer multiples of $k^{-2}$ and that each has length $2 \psi_{k}^{\prime}$.

Assume now that $k^{-2} \leq m^{-2} \leq|J|$. Then we have $j k^{-2} \leq|J|<(j+1) k^{-2}$ for some positive integer $j$. Thus the set $J_{k}=J \cap I_{k}$ has measure

$$
\left|J_{k}\right|<(j+1) 2 \psi_{k}^{\prime}=\frac{j+1}{j} j k^{-2} 2 \psi_{k} \leq \frac{j+1}{j}|J| 2 \psi_{k} .
$$

Summing over all $k \geq m$ and using the fact that $\frac{j+1}{j} \leq 2$, we obtain the desired bound.

The following will be used when $k \leq m$.

For $s \in \mathbb{R}$ define $\|\beta s\|_{\mathrm{o}}=\operatorname{dist}\left(s, \mathbb{Z}_{\mathrm{o}}\right)$, where $\mathbb{Z}_{\mathrm{O}}$ denotes the set of odd integers.

Proposition 13. Let $\beta_{0}=q / p$ with $q$ even and $p$ odd. Let $0<r<1$ and $\beta \in \mathbb{R}$. Then

$$
\mid\left[\beta k^{2} \|_{\mathrm{o}} \geq \frac{1-r}{p} \quad \text { whenever } \quad\left|\beta-\beta_{0}\right| \leq \frac{r}{p k^{2}}, \quad k \in \mathbb{N}_{\mathrm{o}} .\right.
$$

Proof. First, notice that $p^{-1} \leq\left\|\beta_{0} k^{2}\right\|_{\mathrm{o}} \leq 1$ for all odd integers $k$. Clearly,

$$
\beta k^{2}-n=\beta_{0} k^{2}-n_{0}+\left(\beta-\beta_{0}\right) k^{2}-\left(n-n_{0}\right) .
$$

Using the odd integer $n_{0}$ closest to $\beta_{0} k^{2}$ and the odd integer $n$ closest to $\beta k^{2}$, we get

$$
\left\|\beta k^{2}\right\|_{\mathrm{o}}=\left\|\beta_{0} k^{2}\right\|_{\mathrm{o}}+\left(\beta-\beta_{0}\right) k^{2}-\left(n-n_{0}\right) .
$$


Assume that $\left|\beta-\beta_{0}\right| \leq \frac{r}{p k^{2}}$. If $n \neq n_{0}$, then $\left|n-n_{0}\right| \geq 2$ and thus

$$
\mid\left[\beta k^{2} \|_{\mathrm{o}} \geq\left|n-n_{0}\right|-\left|\beta-\beta_{0}\right| k^{2}-\left.|| \beta_{0} k^{2}\right|_{\mathrm{o}} \geq\left|n-n_{0}\right|-\frac{r}{p}-1 \geq \frac{p-r}{p} .\right.
$$

If $n=n_{0}$, then

$$
\left\|\beta k^{2}\right\|_{\mathrm{o}} \geq \mid\left[\beta_{0} k^{2} \|_{\mathrm{o}}-\left|\beta-\beta_{0}\right| k^{2} \geq \frac{1}{p}-\frac{r}{p}=\frac{1-r}{p} .\right.
$$

In both cases we have (28).

Proof of Lemma 9. The compactness of $L_{\alpha}^{-1}: \mathcal{B}_{\rho} \rightarrow \mathcal{B}_{\rho}$ for almost all $\alpha \in \mathbb{R}$ follows from Corollary 11. In order to prove the remaining part of Lemma 9 , fix an even integer $q$ and odd integers $p, K, N$; all positive. Let $\alpha_{0}=p / q$ and $\beta_{0}=q / p$.

Given $C>0$, to be specified later, choose $m \in \mathbb{N}_{\mathrm{o}}$ larger than $K \vee 4$ and sufficiently large such that

$$
\frac{2 C}{(m-2)^{2}} \leq \frac{7}{8 p}, \quad \beta_{0} m^{2}>2 N+4, \quad m^{2}-4 \alpha_{0}>(m-1)^{2}
$$

We define

$$
\psi_{k}=\frac{2 C}{k(k+2)}, \quad k \in \mathbb{N}_{\mathrm{o}}, \quad k \geq m
$$

and $\psi_{k}=0$ if $k$ is even. The sum $\Psi_{m}$ defined in Proposition 10 is given by

$$
\Psi_{m}=\sum_{k \geq m} \psi_{k}=\frac{C}{m} .
$$

Let $\delta=1 /\left(8 p m^{2}\right)$ and $J=\left[\beta_{0}-4 p \delta, \beta_{0}+4 p \delta\right]$. Then by Proposition 12 we have a bound $\mid\left\lceil\beta k^{2}\right\rfloor \geq \psi_{k}$ for every $k \geq m$ and for every $\beta \in J$ outside some set of measure $32 \mathrm{Cp} \delta / \mathrm{m}$ or less. Define

$$
\psi_{k}=\frac{7}{8 p}, \quad k \in \mathbb{N}_{\mathrm{o}}, \quad k<m
$$

Then (28) with $r=1 / 8$ implies that $\mid\left[\beta k^{2} \|_{\mathrm{o}} \geq \psi_{k}\right.$ for all $k<m$ and for all values of $\beta$ in the interval $J_{m}=\left[\beta_{0}-\delta / 2, \beta_{0}+\delta / 2\right]$.

At this point we have proved that $\mid\left\lceil\beta k^{2} \|_{\mathrm{o}} \geq \psi_{k}\right.$ holds for all $k$ and for every $\beta$ in a subset $J_{m}^{\prime} \subset J_{m}$ of measure at least $\left|J_{m}\right|-32 C p \delta / m=\left|J_{m}\right|(1-32 C p / m)$. To complete the proof of Lemma 9, we will now show that there exists a choice of $C>0$, such that if $m \in \mathbb{N}_{\mathrm{o}}$ satisfies (33), then the bounds (23) hold for every $\beta \in J_{m}^{\prime}$, every odd $n \leq N$, and every odd $k \leq K$.

To be more precise, we first restrict $\beta$ to the interval $B=\left[\beta_{0} / 2,2 \beta_{0}\right]$. Then we choose $C$ in such a way that $\beta C$ is larger than $\varphi_{2}(N) \vee \varphi_{1}(K)$ for all $\beta \in B$, where

$$
\varphi_{1}(k)=\frac{7 \beta k^{2}}{4 p}-\frac{49}{64 p^{2}}, \quad \varphi_{2}(n)=\frac{7 n}{4 p}-\frac{49}{64 p^{2}} .
$$


In addition we require $\beta C \geq 1$. In what follows we always assume that $k$ and $n$ are odd positive integers. We also assume that $\beta \in J_{m}^{\prime}$ so that $\left\|\beta k^{2}\right\|_{\mathrm{o}} \geq \psi_{k}$ for every $k$.

We now estimate the values $\phi(n, k)$ defined in (21). Here $\nu=2$. First, we exploit the fact that $\phi(n, k) \geq \phi(1, k)$. It implies that

$$
\phi(n, k) \geq \phi_{1}(k) \wedge \phi(1, m), \quad \phi_{1}(k)=\inf _{\substack{k \leq x<m \\ y \geq 1}}\left|\beta^{2} x^{4}-y^{2}\right|, \quad k<m,
$$

where we have used the notation $s \wedge t=\min (s, t)$. We start by estimating $\phi(1, m)$. If $x \geq m$, then $\left|\beta x^{2}-y\right| \geq \psi_{x} \geq C x^{-2}$ for all $y$. Thus, by (16) we have

$$
\phi(1, m) \geq \inf _{x \geq m}\left(2 \beta x^{2}-C x^{-2}\right) C x^{-2}=2 \beta C-C^{2} m^{-4} \geq \beta C .
$$

For the last inequality we have used the first condition in (33) and the fact that $\beta C \geq 1$. Now consider $\phi_{1}(k)$. Using that $\left|\beta x^{2}-y\right| \geq \frac{7}{8 p}$ for $x<m$ and applying (16), we have

$$
\phi_{1}(k) \geq \inf _{k \leq x<m}\left(2 \beta x^{2}-\frac{7}{8 p}\right) \frac{7}{8 p}=\varphi_{1}(k), \quad k<m .
$$

Notice that this applies to any $k \leq K$, since we have assumed that $m>K$.

Next, we exploit the fact that $\phi(n, k) \geq \phi(n, 1)$. Denote by $\ell$ the largest odd integer not exceeding $\beta m^{2}$. Then

$$
\phi(n, k) \geq \phi_{2}(n) \wedge \phi(\ell, 1), \quad \phi_{2}(n)=\inf _{\substack{x \geq 1 \\ n \leq y<\ell}}\left|\beta^{2} x^{4}-y^{2}\right|, \quad n<\ell .
$$

We start by estimating $\phi(\ell, 1)$. For every $y \in \mathbb{N}_{0}$, denote by $x_{y}$ the odd integer that minimizes $\left|\beta^{2} x^{4}-y^{2}\right|$. By using the third condition in (33), we find that if $y \geq \ell$, then $x_{y} \geq \sqrt{\alpha y}-2>$ $m-3$. Thus $\psi_{x_{y}} \geq \frac{2 C}{(m-2) m}$ whenever $y \geq \ell$, and

$$
\phi(\ell, 1) \geq \inf _{y \geq \ell}\left(2 \beta x_{y}^{2}-\psi_{x_{y}}\right) \psi_{x_{y}} \geq 2 \beta C-\psi_{x_{\ell}}^{2} \geq \beta C .
$$

Here we have used (16), the first condition in (33), and the fact that $\beta C \geq 1$. Now consider $\phi_{2}(n)$. If $x \geq m$, then $\left|\beta x^{2}-y\right|>1$ for every $y<\ell$. On the other hand, if $x<m$, then $\left|\beta x^{2}-y\right| \geq \frac{7}{8 p}$. In either case, we obtain

$$
\phi_{2}(n) \geq \inf _{n \leq y<\ell}\left(2 y-\frac{7}{8 p}\right) \frac{7}{8 p}=\varphi_{2}(n), \quad n<\ell,
$$

where we have again used (16). Notice that this applies to any $n \leq N$ since the second condition in (33) implies that $\ell>N$.

By our assumption on $C$, we have $\beta C \geq \varphi_{2}(n)$ and $\beta C \geq \varphi_{1}(k)$ for all $n \leq N$ and all $k \leq K$. Combining (38), (39), and (40) we find that $\phi(n, k) \geq \varphi_{1}(k)$ for $n \leq N$ and $k \leq K$. Similarly, combining (41), (42), and (43) we find that $\phi(n, k) \geq \varphi_{2}(n)$ for $n \leq N$ and $k \leq K$. Thus,

$$
\phi(n, k) \geq \varphi_{2}(n) \vee \varphi_{1}(k), \quad n \leq N, \quad k \leq K,
$$

holds for every $m \in \mathbb{N}_{\mathrm{o}}$ satisfying (33), and for every $\beta \in B \cap J_{m}^{\prime}$. Substituting the bound (40) for $\varphi_{1}(k)$ and the bound (43) for $\varphi_{2}(n)$ into the inequality (44), and using (22), we obtain the bound (23). As explained above, this completes the proof of Lemma 9. 
5. Estimates done by the computer. What remains to be proved are Lemma 5 and two analogous lemmas that imply Theorems 2 and 3. We will only describe here the proof of Lemma 5. The other two lemmas are proved similarly, and we refer the reader to the supplementary material for complete details.

Lemma 5 involves the choice of parameters $\left(\rho, u_{0}, A, K, \delta, \varepsilon\right)$ for each value of $\kappa \in Q_{2}$. The precise value of each parameter, as well as the complete set $Q_{2}$, is given in the supplementary material. To be more precise, only the set $Q_{2}$ and the domain parameter $\rho$ for each $\kappa \in Q_{2}$ are specified explicitly. The other parameters are computed as specified by our programs. In particular, the approximate fixed point $u_{0}$ is first guessed and then improved by iterating a numerical version of $\mathcal{N}_{\kappa}$. The operator $A: \mathcal{B}_{\rho} \rightarrow \mathcal{B}_{\rho}$ is the approximate inverse of a numerical approximation for the operator $\mathrm{I}-D \mathcal{F}_{\kappa}\left(u_{0}\right)$. The constants $K, \delta, \varepsilon$ are determined a posteriori to satisfy (12). At the end, we verify that $\varepsilon+K \delta<\delta$ and that there exists $r \geq \delta\|A\|_{\rho}$ such that every function $u \in B_{r}\left(u_{0}\right)$ is of type $(1,1)$ and satisfies $\left|u_{1,1}\right|>\sqrt{2|1-\alpha|}$.

This leaves the task of estimating the two norms in (12) and the operator norm of $A$. The norm of the operator $\mathcal{L}=D \mathcal{N}_{\alpha}(h)$ for $h \in B_{\delta}(0)$ is estimated by using the inequality (15). So all we need are bounds on the function $\mathcal{N}_{\alpha}(0)=\mathcal{F}_{\alpha}\left(u_{0}\right)-u_{0}$, on $\mathcal{L} P_{n, k}$ for $n \leq N$ and $k \leq K$, and on $\mathcal{L} E_{n, k}$ for $n \leq 2 N$ and $k \leq 2 K$. Here $E_{n, k}$ denotes a function in $\mathcal{A}_{n, k}^{\mathrm{o}}$ which is unknown except for a bound on its norm. The operator norm of $A$ is estimated similarly. These are standard tasks in many computer-assisted proofs, including those in [5].

At this level, our techniques are similar to the techniques used in [5] to find solutions for the boundary value problem $-\Delta u=w u^{3}$ on the unit square. The function spaces are in fact the same. As far as estimates are concerned, the main difference is that we now have the operator $L_{\alpha}^{-1}$ instead of the inverse Laplacian. This is where we use the bounds described in section 3. But at the level of enclosures (representable sets in $\mathcal{A}_{n, k}$ ) and data types used to represent such enclosures, we use the same methods as in [5]. Thus, we refer the reader to [5] for a description of the basic principles.

The main goal of such a description is to simplify the reading of our computer programs [5]. The source code of these programs contains the details of how the remaining part of the proof is organized. Our code is written in the programming language Ada [1] and was compiled using a public version of the GCC/GNAT compiler [16]. By running the resulting machine code, the computer verifies the inequalities necessary to complete the proofs of Lemma 5 and Theorem 4 .

\section{REFERENCES}

[1] Ada Reference Manual, ISO/IEC 8652:2012(E), Ada Conformity Assessment Authority, Madison, WI, 2012, http://www.ada-auth.org/arm.html.

[2] G. Arioli and F. Gazzola, Torsional instability in suspension bridges: The Tacoma Narrows Bridge case, Comm. Nonlinear Sci. Numer. Simul., 42 (2017), pp. 342-357.

[3] G. Arioli And F. Gazzola, On a nonlinear nonlocal hyperbolic system modeling suspension bridges, Milan J. Math., 83 (2015), pp. 211-236.

[4] G. Arioli And H. Koch, Some symmetric boundary value problems and non-symmetric solutions, J. Differential Equations, 259 (2015), pp. 796-816.

[5] G. ARIOli ANd H. Koch, Non-symmetric low-index solutions for a symmetric boundary value problem, J. Differential Equations, 252 (2012), pp. 448-458. 
[6] G. Arioli And H. Koch, Computer-assisted methods for the study of stationary solutions in dissipative systems, applied to the Kuramoto-Sivashinsky equation, Arch. Ration. Mech. Anal., 197 (2010), pp. 1033-1051.

[7] G. Arioli And H. КосH, Integration of dissipative partial differential equations: A case study, SIAM J. Appl. Dyn. Syst., 9 (2010), pp. 1119-1133, https://doi.org/10.1137/10078298X.

[8] M. BerTi, Nonlinear Oscillations of Hamiltonian PDEs, Birkhäuser Boston, Inc., Boston, MA, 2007.

[9] R. Castelli, M. Gameiro, and J.-P. Lessard, Rigorous Numerics for Ill-posed PDEs: Periodic Orbits in the Boussinesq Equation, preprint, 2015, https://arxiv.org/abs/1509.08648.

[10] J. CYRANKA AND P. ZGLICZYŃSKI, Existence of globally attracting solutions for one-dimensional viscous Burgers equation with nonautonomous forcing - A computer assisted proof, SIAM J. Appl. Dyn. Syst., 14 (2015), pp. 787-821, https://doi.org/10.1137/14096699X.

[11] L. H. Eliasson, B. GréBert, and S. Kuksin, KAM for the Nonlinear Beam Equation 1: SmallAmplitude Solutions, preprint, 2015, https://arxiv.org/abs/1412.2803.

[12] J.-L. Figueras And R. DE la Llave, Numerical Computations and Computer Assisted Proofs of Periodic Orbits of the Kuramoto-Sivashinsky Equation, preprint, 2016, https://arxiv.org/abs/1605.01085.

[13] M. Gameiro And J.-P. Lessard, A Posteriori Verification of Invariant Objects of Evolution Equations: Periodic Orbits in the Kuramoto-Sivashinsky PDE, preprint, 2016. Available online at http://archimede.mat.ulaval.ca/jplessard/Publications_files/KS_v10.pdf.

[14] G. Gentile, V. Mastropietro, and M. Procesi, Periodic solutions for completely resonant nonlinear wave equations with Dirichlet boundary conditions, Comm. Math. Phys., 256 (2005), pp. 437-490.

[15] G. Gentile And M. Procesi, Periodic solutions for a class of nonlinear partial differential equations in higher dimension, Comm. Math. Phys., 289 (2009), pp. 863-906.

[16] GNAT: A Free, High-Quality, Complete Compiler for Ada, Integrated into the GCC Compiler System, Free Software Foundation, Inc., Boston, MA, 2014, https://www.gnu.org/software/gnat/.

[17] W. S. Hall, On the existence of periodic solutions for the equations $D_{t t} u+(-1)^{p} D_{x}^{2 p} u=\varepsilon f(\cdot, \cdot, u), \mathrm{J}$. Differential Equations, 7 (1970), pp. 509-526.

[18] W. S. Hall, Periodic solutions of a class of weakly nonlinear evolution equations, Arch. Ration. Mech. Anal., 39 (1970), pp. 294-322.

[19] S. Lang, Elliptic Curves: Diophantine Analysis, Springer-Verlag, Berlin, 1978.

[20] C. LEe, Periodic solutions of beam equations with symmetry, Nonlinear Anal., 42 (2000), pp. 631-650.

[21] J. Q. Liu, Free vibrations for an asymmetric beam equation, II, Nonlinear Anal., 56 (2004), pp. 415-432.

[22] J. Q. LiU, Free vibrations for an asymmetric beam equation, Nonlinear Anal., 51 (2002), pp. 487-497.

[23] V. Mastropietro And M. Procesi, Lindstedt series for periodic solutions of beam equations with quadratic and velocity dependent nonlinearities, Commun. Pure Appl. Anal., 5 (2006), pp. 1-28.

[24] P. J. McKenna, F. Pacella, M. Plum, and D. Roth, A uniqueness result for a semilinear elliptic problem: A computer-assisted proof, J. Differential Equations, 247 (2009), pp. 2140-2162.

[25] M. T. Nakao, Y. Watanabe, N. Yamamoto, T. Nishida, and M.-N. Kim, Computer assisted proofs of bifurcating solutions for nonlinear heat convection problems, J. Sci. Comput., 43 (2010), pp. 388-401.

[26] F. Pacella, M. Plum, And D. Rütters, A Computer-Assisted Existence Proof for Emden's Equation on an Unbounded L-shaped Domain, preprint, 2016, https://arxiv.org/abs/1601.01543.

[27] P. H. Rabinowitz, On nontrivial solutions of a semilinear wave equation, Ann. Scuola Norm. Sup. Pisa Cl. Sci., 8 (1981), pp. 647-657.

[28] P. H. Rabinowitz, Free vibration for a semilinear wave equation, Comm. Pure Appl. Math., 31 (1978), pp. 31-68.

[29] W. M. Schmidt, Metrical theorems on fractional parts of sequences, Trans. Amer. Math. Soc., 110 (1964), pp. $493-518$.

[30] P. ZGLICZyŃski, Rigorous numerics for dissipative PDEs III. An effective algorithm for rigorous integration of dissipative PDEs, Topol. Methods Nonlinear Anal., 30 (2010), pp. 197-262.

[31] P. Zgliczý́ski, Rigorous numerics for dissipative partial differential equations II. Periodic orbit for the Kuramoto-Sivashinsky PDE. A computer-assisted proof, Found. Comput. Math., 4 (2004), pp. 157185.

[32] P. Zgliczyński And K. Mischaikow, Rigorous numerics for partial differential equations: The Kuramoto-Sivashinsky equation, Found. Comput. Math., 1 (2001), pp. 255-288. 